\title{
Augmented reality guided osteotomy in hallux Valgus correction
}

\author{
Arnd Fredrik Viehöfer ${ }^{1 *} \mathbb{D}$, Stephan Hermann Wirth ${ }^{1,2}$, Stefan Michael Zimmermann ${ }^{1}$, Laurenz Jaberg ${ }^{1}$, \\ Cyrill Dennler', Philipp Fürnstahl ${ }^{2}$ and Mazda Farshad ${ }^{1}$
}

\begin{abstract}
Background: An optimal osteotomy angle avoids shortening of the first metatarsal bone after hallux valgus surgery and therefore reduces the risk of transfer-metatarsalgia. The purpose of the present ex-vivo study was to investigate whether augmented reality (AR) would improve accuracy of the distal osteotomy during hallux valgus surgery.

Methods: Distal osteotomies of the first metatarsals were performed on a foot model by two surgeons with different levels of surgical experience each with $(A R, n=15 \times 2)$ or without (controls, $n=15 \times 2)$ overlay of a hologram depicting an angle of osteotomy perpendicular to the second metatarsal. Subsequently, the deviation of the osteotomy angle in the transverse plane was analyzed.

Results: Overall, AR decreased the extent of deviation and the AR guided osteotomies were more accurate $\left(4.9 \pm 4.2^{\circ}\right)$ compared to the freehand cuts $\left(6.7 \pm 6.1^{\circ}\right)$ by tendency $(p=0.2)$. However, while the inexperienced surgeon performed more accurate osteotomies with AR with a mean angle of $6.4 \pm 3.5^{\circ}$ compared to freehand $10.5 \pm 5.5^{\circ}(p=0.02)$, no significant difference was noticed for the experienced surgeon with an osteotomy angle of around $3^{\circ}$ in both cases.

Conclusion: This pilot-study suggests that AR guided osteotomies can potentially improve accuracy during hallux valgus correction, particularly for less experienced surgeons.
\end{abstract}

Keywords: Augmented reality, First ray shortening, Transfermetatarsalgia, Hallux valgus

\section{Background}

Hallux valgus deformity is a one of the most common deformities of the foot [1]. Once conservative measures have failed, distal first metatarsal (MT I) osteotomies (e.g. ReveL) are well established techniques to correct hallux valgus deformities [2-6]. The principal technique of these operations is to osteotomize the MT I and to shift the distal part laterally along the plumb line of the second metatarsal in the transverse plane [4]. Accurate performance of the osteotomy is mandatory to avoid additional shortening of the MT I. Geometrical analysis has shown that a posterior deviation of only $10^{\circ}$ shortens

\footnotetext{
* Correspondence: arnd.viehoefer@balgrist.ch

'Department of Orthopaedics, Balgrist University Hospital, Forchstrasse 340, 8008 Zürich, Switzerland

Full list of author information is available at the end of the article
}

the MT I by $5 \mathrm{~mm}$ and might therefore lead to transfer metatarsalgia postoperatively. Various technologies have been employed to increase the accuracy in implementation of the optimal planning, but their clinical application has been challenged by increased surgical time, exposure, as well as need for additional costly infrastructure or slow learning curves. A recent promising technological advancement is augmented reality (AR), making a superimposition of a hologram to reality possible, providing additional information to the performing surgeon. This can be realized by a semitransparent mirror which allows the surgeon to see the generated images projected onto real objects in so called "Video SeeThrough Head-Mounted Display "(VSTHMD) systems. The first AR VSTHMD was built in 1968 by Sutherland [7]. More recent improvements in AR allow to display generated images in real time on small head mounted displays (e.g.

(c) The Author(s). 2020 Open Access This article is licensed under a Creative Commons Attribution 4.0 International License, which permits use, sharing, adaptation, distribution and reproduction in any medium or format, as long as you give appropriate credit to the original author(s) and the source, provide a link to the Creative Commons licence, and indicate if changes were made. The images or other third party material in this article are included in the article's Creative Commons licence, unless indicated otherwise in a credit line to the material. If material is not included in the article's Creative Commons licence and your intended use is not permitted by statutory regulation or exceeds the permitted use, you will need to obtain permission directly from the copyright holder. To view a copy of this licence, visit http://creativecommons.org/licenses/by/4.0/ The Creative Commons Public Domain Dedication waiver (http://creativecommons.org/publicdomain/zero/1.0/) applies to the data made available in this article, unless otherwise stated in a credit line to the data. 
Hololens $^{\mathrm{Tm}}$ ), making AR now feasible for use in the operation theatre. AR has been evaluated in several surgical fields [8-13], but to our knowledge, no study has yet evaluated the use of AR in foot surgery.

The purpose of the present ex-vivo study was to investigate whether overlaying a hologram to reality would improve accuracy of the distal osteotomy for hallux valgus surgery.

We hypothesized that overlaying a hologram will increase the accuracy of distal MT I osteotomy, especially when carried out by inexperienced surgeons.

\section{Methods}

\section{The foot models}

The experiments were performed on a foot dummy consisting of a polyamide forefoot skeleton with an exchangable first metatarsal bone (MT I) with a soft tissue cover, made of medical silicone (Fig. 1).

To build the 3 dimensional forefoot skeleton, a CT scan (Philips Brilliance $40 \mathrm{CT}$, Philips Healthcare, The Netherlands) from a cadaveric foot with hallux valgus deformity was conducted under axial load of $350 \mathrm{~N}$ in a custom made clamping system. The data of the 3 dimensional model of the foot were segmented and implemented in the preoperative planning software CASPA (version 4.18, Balgrist CARD AG, Zurich, Switzerland). The foot showed a moderate hallux valgus deformity with an intermetatarsal angle (IMA) of $15^{\circ}$. In order to obtain three different hallux valgus manifestations (slight, moderate and severe), the MT I was rotated parallel to the plantar plate to an IMA of $18^{\circ}$ (severe hallux valgus) and $13^{\circ}$ (slight hallux valgus) through the center of the sphere defined by the proximal articular surface of the MT I. A pluggable connector was

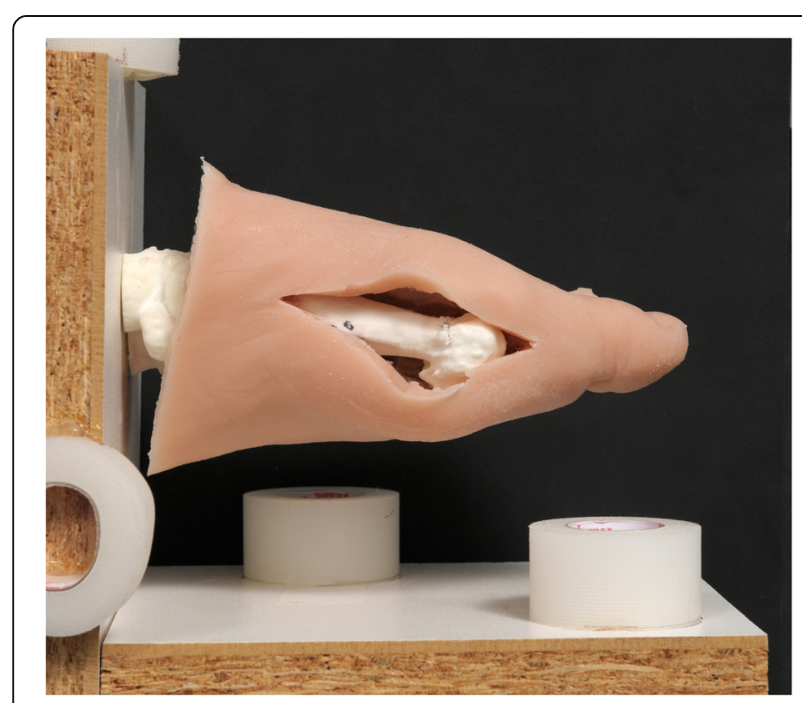

Fig. 1 Foot dummy. The approach through the silicon reveals shows the exchangeable polyamide MT I incorporated in the model between the MT I and the medial cuneiform bone to permit exchange of the MT I after completion of an osteotomy. The polyamide skeleton was printed with 60 replaceable MT I (with 20 slight, 20 moderate and 20 severe hallux valgus manifestations) using selective laser sintering [14].

The soft tissue cover of the foot dummy model was generated by using a plaster cast mold of the forefoot and midfoot of the cadaver. The cast was lined with a dividing layer (Body Double, SmoothOn, Macungie PA, USA) and filled with medical silicon (Elastosil, Wacker, Riemerling, Germany). The polyamide skeleton model was subsequently immerged in the silicone before the silicon had cured.

A dorsomedial approach through the silicone cover to the MT I was performed (AFV and SMZ). Finally, the model was fixed to a wooden frame with 3 dimensional markers attached to it for facilitated alignment of the hologram.

For the augmented reality (AR) experiment, a cutting plane perpendicular to the axis of the second metatarsal bone and perpendicular to the plantar plate was integrated in the model to serve as cutting guide in the experiments (Fig. 2). The model for slight, moderate, and severe hallux valgus, including the cutting plane and the model of the frame and its markers, were then transferred to the head mounted device (HMD) (Hololens ${ }^{\mathrm{Tm}}$, Microsoft Corporation, Redmond, WA 98052-6399, USA)).

\section{Experiments}

The distal osteotomy of the MT I was performed using an oscillating saw blade (Sodem Systems, Geneva, Switzerland). The cuts were alternately carried out freehand or with an overlaid hologram wearing the HMD (Fig. 3). They were performed by an experienced surgeon in foot and ankle

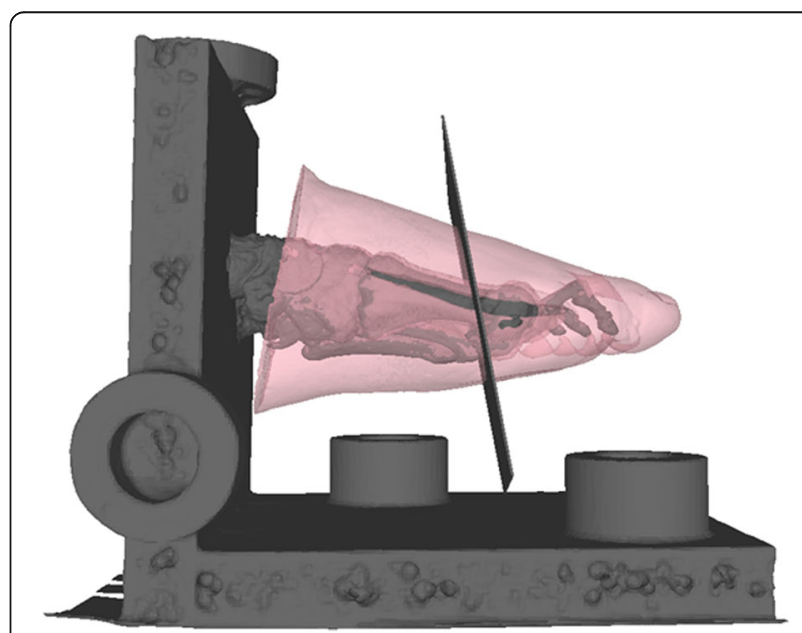

Fig. 23 dimensional model of the foot: The hologram model projected in the experiments. The plane guides an osteotomy perpendicular to the second metatarsal 


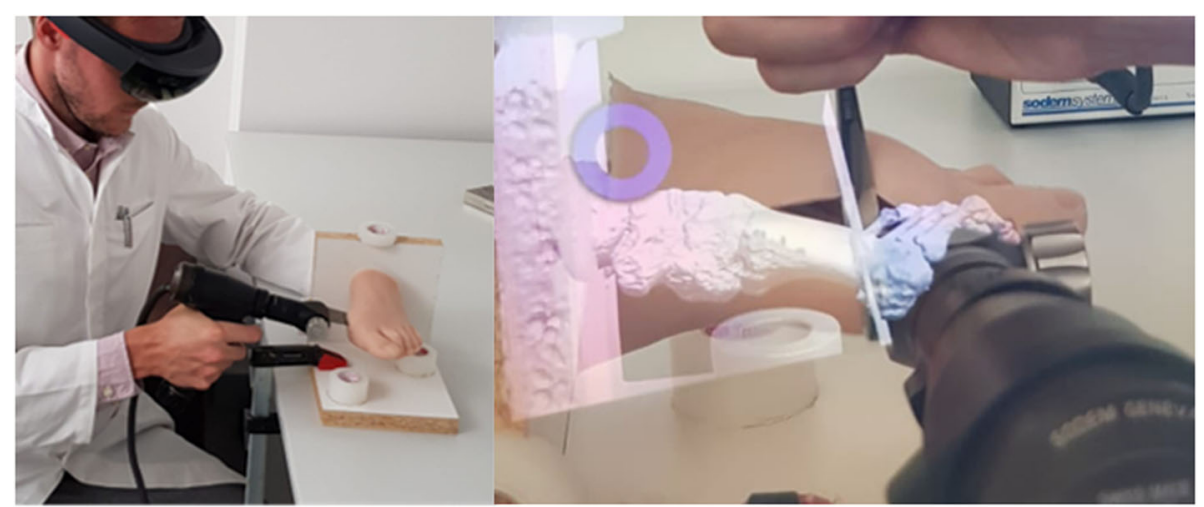

Fig. 3 Setup of the experiments with augmented reality. Left: Surgeon performing the osteotomy wearing the Hololens ${ }^{\text {TM. }}$ Right: foot model with overlaying hologram

surgery and an unexperienced surgeon. The experienced surgeon (SMZ) had worked as a consultant in the foot and ankle department for 2 years and performed hallux valgus surgery on a daily routine. The inexperienced surgeon was a resident (LJ) who had no prior experience in hallux valgus surgery at the time of the experiments. The experiments were randomized concerning freehand or AR cuts and the manifestation of the hallux valgus deformity. Each surgeon performed 15 osteotomies with HololensTM and 15 freehand osteotomies with randomized degree of deformity. In an attempt to avoid adaptation to the setting, a maximum of 6 experiments was set for each surgeon per day.

\section{Analysis}

A second skeletal model (Fig. 4) was printed for analysis without the first metatarsal but a plane perpendicular to the plantar plane and parallel to the axis of the second metatarsal. The osteotomized first metatarsals of the experiments could be connected to the analysis skeleton model through a plug. A picture of each MT I osteotomy was taken perpendicular to the plantar plate and centered over the distal first metatarsal with a camera

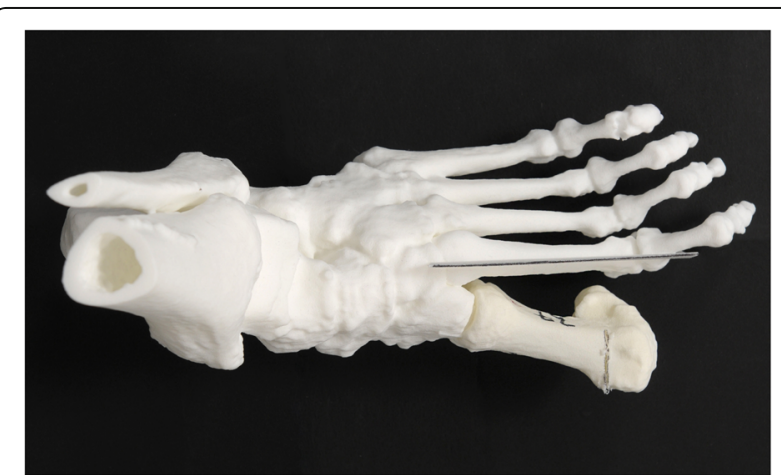

Fig. 4 Skeletal model for analysing the osteotomie angle in relation to axis of the second metatarsal (black line). An osteotomised first metatarsal is shown exemplary
(Nikon, Tokyo, Japan). The angle between the axis of the second metatarsal and the osteotomy was measured using the measurement tool in Synedra (Synedra Schweiz AG, Dübendorf, Switzerland). The deviation in other planes than the plantar plane were not considered as they don't account for changes in MT I length postoperatively. The measurements were carried out by two independent observers blinded to the surgical method and surgeon that performed the osteotomy (LJ and AFV). The statistical analysis was performed using SPSS (IBM, Armonk, NY, USA). The Student's T test for independent samples was used to determine whether the differences were significant $(p<0.05)$, assuming normal distribution of data. Cronbach's Alpha was used to measure internal consistency (inter observer correlation). A post hoc power analysis was performed with G*Power (Heinrich-Heine-University, Düsseldorf, Germany).

\section{Source of funding}

ResOrtho Foundation, University Hospital Balgrist. Funding source did not play a role in this investigation.

\section{Results}

Overall, the mean deviation between the osteotomy plane and the target plane perpendicular to the second metatarsal was $4.9 \pm 4.2^{\circ}$ in anterior direction (i.e. from proximal medial to distal lateral) with help of AR, and $6.7 \pm 6.1^{\circ}$ in anterior direction for freehand osteotomies. The difference between the osteotomy angle between freehand performance and experiments with AR was not significant if the combined average performances of both surgeons were considered ( $p=0.2)$ (Fig. 5). However, AR decreased the extent of deviation with a smaller standard deviation (regular $6.1^{\circ}$, AR $4.2^{\circ}$ ) and a smaller range (regular $25^{\circ}, \mathrm{AR} 18^{\circ}$ ).For the more experienced foot surgeon, the use of AR did not increase the accuracy of distal osteotomies significantly overall. The mean deviation from the plumb line of the osteotomy to the second 


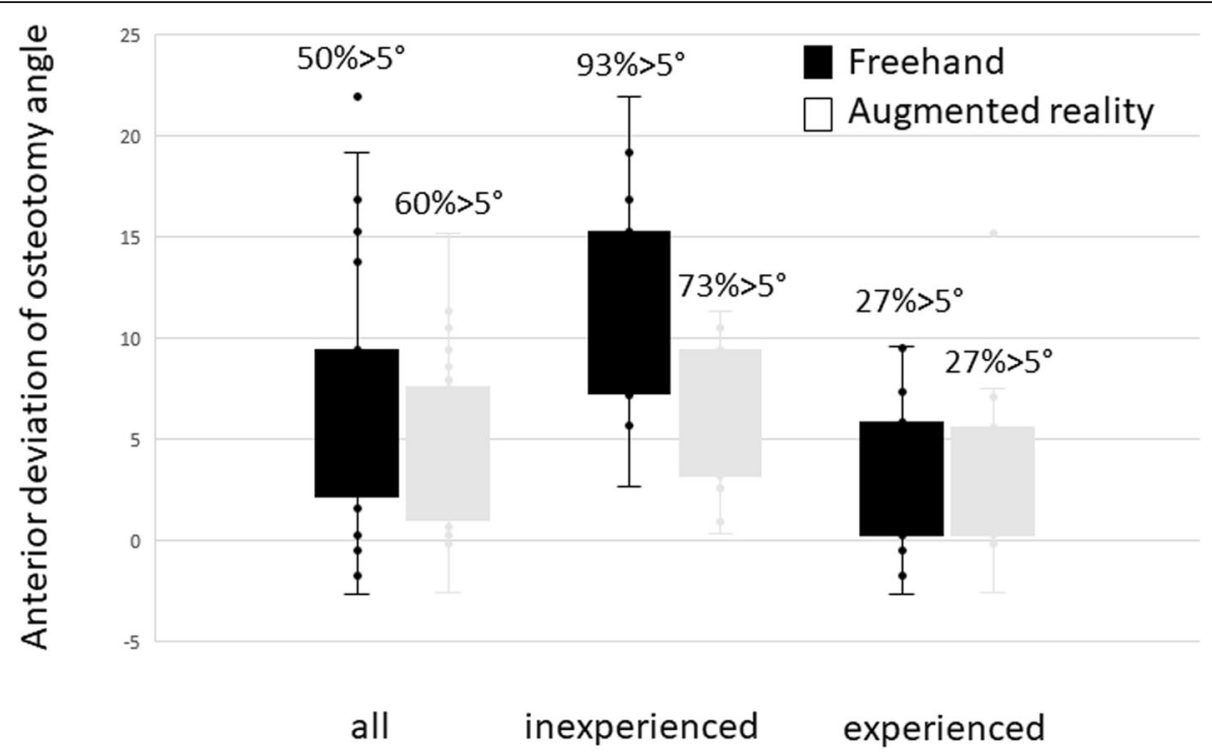

Fig. 5 Deviation of osteotomy angle. Outliers $>5^{\circ}$ are given in percentage for each setup. Left: Osteotomy angle for both surgeons. The results for AR (mean angle $4.1^{\circ}$ ) and freehand osteotomies (mean angle $6.1^{\circ}$ ) did not differ significantly $(p=0.2$ ), but the variability of imprecision is less with AR-support. Middle: Osteotomy angle for the experienced surgeon. The results for the AR (mean angle $3.4^{\circ}$ ) and freehand (mean angle $2.8^{\circ}$ ) osteotomies did not differ significantly $(p=0.2$ ). right: Osteotomy angle for the inexperienced surgeon. The osteotomy angle for the AR (mean angle $6.4^{\circ}$ ) differed significantly $(p=0.02$ ) less from the plane perpendicular to the second metatarsal than the freehand osteotomies (mean angle $10.5^{\circ}$. Also the variability of imprecision is higher without AR-support

metatarsal was $3.4 \pm 4.3^{\circ}$ (range 18) with AR and $2.8 \pm$ $3.8^{\circ}$ for the freehand osteotomies (range 11, (Fig. 5). For the less experienced surgeon, the osteotomies with AR were significantly $(p=0.02)$ more accurate with an angle of $6.4 \pm 3.5^{\circ}$ (range 19) compared to the freehand osteotomies with an angle of $10.5 \pm 5.5^{\circ}$ (range 12, Fig. 5). The maximum deviation for the less experienced surgeon was $22^{\circ}$ for the freehand and $11^{\circ}$ for the AR supported osteotomies.

Although the more experienced surgeon also achieved better results with AR-support than the less experienced surgeon, this finding was not significant enough in our series of experiments $(P=0.051)$. There was no significant difference in the precision of osteotomy angles for the different simulated manifestation of the deformity Fig. 6.

The reported mean deviations imply an anterior deviation in regard to the plumb line of the second metatarsal bone. A posterior deviation was only observed in $10 \%$ (6/60. AR 3, regular 3) of our cases.

The analysis showed excellent inter-observer reliability with a Cronbach's Alpha of 0.978 .

The post hoc power analysis stated a power $1-\beta$ of 0.92 for an effect size $d$ of 0.8 and a power of 0.61 for an effect size of $d=0.5(\alpha=0.05)$.

\section{Discussion}

The aim of the present study was to investigate whether AR improves accuracy of the osteotomy angle in distal hallux valgus surgery. We hypothesized that overlaying a hologram helps surgeons perform the osteotomy more accurately, especially when inexperienced. We present the first report on potential merits of a promising new technology (AR) in a randomized ex-vivo study, and report the accuracy of the distal osteotomy during hallux valgus surgery with and without the support of this new technology with different levels of surgical experience.

When viewing the results for the two surgeons individually, the experienced surgeon did not perform the osteotomies more accurately with help of AR. The inexperienced surgeon however obtained better results when using AR technology. The accuracy of the osteotomy angle improved significantly for the osteotomies with $\mathrm{AR}(p=0.02)$ by $4^{\circ}$. Without $\mathrm{AR}$, the osteotomy revealed a mean deviation of $10.5^{\circ}$ from the plane perpendicular to the second metatarsal. In accordance, the range of accuracy was much larger without AR-support. This was also true if the results of both surgeons were considered. The here observed important differences outline the power of AR guided osteotomies to decrease the learning curve of less experienced surgeons. This finding is in line with the results of other types of navigated surgery [15]. A correct osteotomy angle can avoid shortening of the first metatarsal bone after hallux valgus surgery and therefore reduce the risk of transfer-metatarsalgia. According to geometrical considerations, a posterior deviation of $10^{\circ}$ to the plane perpendicular to the second metatarsal may shorten the MT I by up to $5 \mathrm{~mm}$ for 


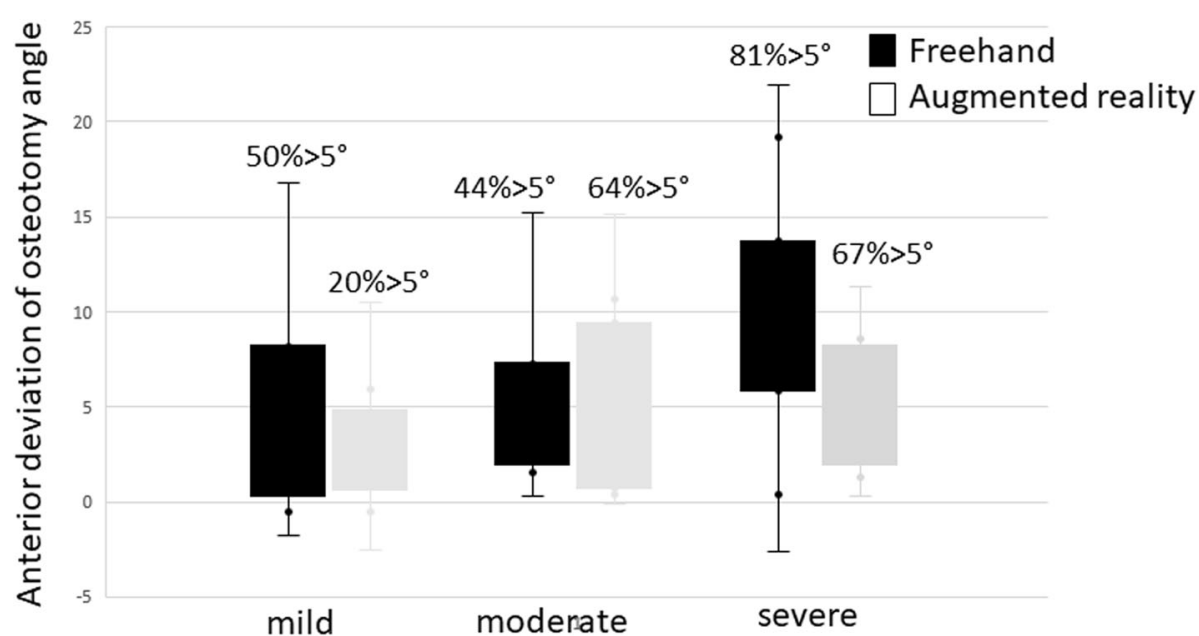

Fig. 6 Results for freehand and Hololens ${ }^{T M}$ osteotomies for different hallux valgus manifestations. Outliers $>5^{\circ}$ are given in percentage for each setup. Left: mild hallux valgus. Middle: moderate hallux valgus. Right: severe hallux valgus

correction of severe hallux valgus. With the current technique of free-hand osteotomies, an unwanted variability in accuracy is observed. The improved osteotomy angle with AR-support for the inexperienced surgeon would reduce shortening of the MT I by $20 \%$ to around $4 \mathrm{~mm}$ if the divergence would be in the posterior direction. This would even be more pronounced if the differences between the expected shortening for the maximum deviation of the osteotomy angle $\left(22^{\circ}\right.$ freehand and $\left.11^{\circ} \mathrm{AR}\right)$ are analyzed: For the inexperienced surgeon, the difference would be 3 $\mathrm{mm}$ or $38 \%$ ( $8 \mathrm{~mm}$ freehand and $5 \mathrm{~mm}$ AR of MT I shortening, respectively). Although the correlation of amount of shortening of the MT I to transfer-metatarsalgia might not be direct, recent studies have shown an increased risk for metatarsalgia in patients with shortening of as little as $2 \mathrm{~mm}$ [16]. For an anterior deviation of the osteotomy angle, as observed in the present study, the differences in length become distinctly smaller. Of notice, we routinely record a tendency toward posterior deviation for freehand cuts in our clinical observation in contrast to the anterior deviation observed in the present study. This could be explained due to the model set up, which is the main limitation of this ex vivo experiment.

The power analysis showed a sufficient power for an effect size of 0.8 , but not for an effect size of 0.5 . The observed tendency towards a higher accuracy for AR performed osteotomies for both surgeons might become statistically significant for higher sample sizes. We believe an effect size of 0.8 to be sufficient, as small deviations in the osteotomy angle are not clinically significant.

Furthermore, a higher sample size in this experimental set up also risks that the freehand osteotomies are performed more accurately due to a learning curve resulting from the AR guided osteotomies.
Although a lot of effort was made to create a realistic foot model, artificial bones with a soft tissue cover were used. It is likely that the alignment of the hologram, although simplified by the frame of the foot, is a source of error. Further sources of error include precision of the augmented reality equipment, and performing the osteotomy guided by the hologram. Finally, the present study cannot quantitively assess the influence of each source of error Advancements of the holographic technology already allow a better alignment of the hologram.

\section{Conclusion}

Considering the experimental setup, we conclude that AR is a powerful technology with the potential to improve accuracy in hallux valgus correction, particularly for less experienced surgeons. Following the here presented first report of use of AR in foot surgery, clinical studies are necessary to verify whether this promising technology will improve the outcome following hallux valgus surgery.

\section{Abbreviations}

AR: Augmented reality; HMD: Head mounted device; IMA: Intermetatarsal angle; MT I: First metatarsal bone; VSTHMD: Video see-through head-mounted display

\section{Acknowledgments}

We want to thank Michael Hecht of the CARD team for his help in preparation of the foot model.

\section{Authors' contributions}

SHW: research design, interpretation of data, revising the manuscript, approval of the submitted and final versions. SMZ: acquisition of data, writing manuscript, approval of the submitted and final versions. IJ: execution of the experiments, acquisition and analysis of data. CD: research design, analysis of data, approval of the submitted and final versions. PF: research design, revising manuscript, approval of the submitted and final versions. MF: research design, interpretation of data, revising manuscript, approval of the submitted and final versions. AFV: research design, acquisition, analysis and interpretation of data, writing manuscript, approval of the submitted and final versions. 


\section{Funding}

This research was funded by the Balgrist Foundation.

\section{Availability of data and materials}

The datasets used and/or analysed during the current study are available from the corresponding author on reasonable request.

\section{Ethics approval and consent to participate}

Approval for the study was provided by the local Ethics Committee of Zurich and a consent to participate was given in written form.

\section{Consent for publication}

Not applicable.

\section{Competing interests}

One author (PF) is shareholder of the Balgrist CARD AG - a company producing PSI for osteotomies.

\section{Author details}

'Department of Orthopaedics, Balgrist University Hospital, Forchstrasse 340, 8008 Zürich, Switzerland. ${ }^{2}$ Computer-Assisted Research and Development Group, Balgrist University Hospital, Zurich, Switzerland.

Received: 21 February 2020 Accepted: 27 May 2020

Published online: 17 June 2020

\section{References}

1. Roddy E, Zhang WDoherty M. Prevalence and associations of hallux valgus in a primary care population. Arthritis Rheum. 2008;59:857-62.

2. Potenza V, Caterini R, Farsetti P, Forconi F, Savarese E, Nicoletti SIppolito E. Chevron osteotomy with lateral release and adductor tenotomy for hallux valgus. Foot Ankle Int. 2009;30:512-6.

3. Cassinelli SJ, Herman RHarris TG. Distal metatarsal osteotomy for moderate to severe hallux Valgus. Foot Ankle Int. 2016;37:1137-45.

4. Helmy N, Vienne P, von Campe AEspinosa N. Treatment of hallux valgus deformity: preliminary results with a modified distal metatarsal osteotomy. Acta Orthop Belg. 2009;75:661-70.

5. Biz C, Corradin M, Petretta IAldegheri R. Endolog technique for correction of hallux valgus: a prospective study of 30 patients with 4-year follow-up. J Orthop Surg Res. 2015;10:102.

6. Biz C, Fosser M, Dalmau-Pastor M, Corradin M, Roda MG, Aldegheri RRuggieri P. Functional and radiographic outcomes of hallux valgus correction by mini-invasive surgery with Reverdin-Isham and akin percutaneous osteotomies: a longitudinal prospective study with a 48month follow-up. J Orthop Surg Res. 2016;11:157.

7. Sutherland IE. A head-mounted three dimensional display. Proc Fall Joint Comput Conf. 1968:757-64. https://doi.org/10.1145/1476589.1476686.

8. Badiali G, Ferrari V, Cutolo F, Freschi C, Caramella D, Bianchi AMarchetti C. Augmented reality as an aid in maxillofacial surgery: validation of a wearable system allowing maxillary repositioning. J Craniomaxillofac Surg. 2014;42:1970-6

9. Cutolo F, Meola A, Carbone M, Sinceri S, Cagnazzo F, Denaro E, Esposito N Ferrari MFerrari V. A new head-mounted display-based augmented reality system in neurosurgical oncology: a study on phantom. Comput Assist Surg (Abingdon). 2017;22:39-53.

10. Dutra-Medeiros M, Nascimento J, Henriques J, Barrao S, Fernandes-Fonseca A, Aguiar-Silva N, Moura-Coelho NAgoas V. Three-dimensional headmounted display system for ophthalmic surgical procedures. Retina. 2017; 37:1411-4.

11. Kihara K, Fujii Y, Masuda H, Saito K, Koga F, Matsuoka Y, Numao NKojima K. New three-dimensional head-mounted display system, TMDU-S-3D system, for minimally invasive surgery application: procedures for gasless single-port radical nephrectomy. Int J Urol. 2012;19:886-9.

12. Meola A, Cutolo F, Carbone M, Cagnazzo F, Ferrari MFerrari V. Augmented reality in neurosurgery: a systematic review. Neurosurg Rev. 2017;40:537-48.

13. Wu JR, Wang ML, Liu KC, Hu MHLee PY. Real-time advanced spinal surgery via visible patient model and augmented reality system. Comput Methods Prog Biomed. 2014;113:869-81.

14. Schweizer A, Furnstahl PNagy L. Three-dimensional correction of distal radius intra-articular malunions using patient-specific drill guides. J Hand Surg Am. 2013;38:2339-47.
15. Jenny JYPicard F. Learning navigation - learning with navigation. a review. SICOT J. 2017;3:39.

16. Zhang FQ, Pei BY, Wei ST, Zhao HT, Li ZY, Gao JGZhang YZ. Correlative study between length of first metatarsal and transfer metatarsalgia after osteotomy of first metatarsal. Zhonghua Yi Xue Za Zhi. 2013;93:3441-4.

\section{Publisher's Note}

Springer Nature remains neutral with regard to jurisdictional claims in published maps and institutional affiliations.
Ready to submit your research? Choose BMC and benefit from:

- fast, convenient online submission

- thorough peer review by experienced researchers in your field

- rapid publication on acceptance

- support for research data, including large and complex data types

- gold Open Access which fosters wider collaboration and increased citations

- maximum visibility for your research: over $100 \mathrm{M}$ website views per year

At BMC, research is always in progress.

Learn more biomedcentral.com/submissions 